\title{
Pitfalls of Contrast Enhanced Ultrasound (CEUS) in determination of breast tumor biological dignity
}

\author{
V. LEHOTSKA ${ }^{1,2}$, K. RAUOVA ${ }^{1,2}$, L. VANOVCANOVA ${ }^{1,2}$ \\ ${ }^{1} 2^{\text {nd }}$ Radiology Department, Faculty of Medicine, Comenius University in Bratislava, Slovakia; ${ }^{2}$ St. Elizabeth's Cancer Institute in Bratislava, \\ Slovakia \\ ${ }^{*}$ Correspondence: viera.lehotska@ousa.sk
}

Received January 16, 2017 / Accepted April 20, 2017

\begin{abstract}
The aim of this prospective study was to identify characteristics of breast lesions on contrast enhanced ultrasound (CEUS) that can be a source of diagnostic ambiguity and cause under- or overestimation of examined breast lesions.

215 women (range 23-76y., mean age 53.16 y) with 215 breast lesions were examined by B-mode ultrasound, followed by CEUS and core biopsy. CEUS parameters: degree of opacification, character of washout and preliminary CEUS result (suspicious, non-suspicious, uncertain) were subsequently correlated with histopathological outcomes. Observed qualitative variables, CEUS results and their analysis with respect to histological type were evaluated using Pearson $\chi^{2}$ square test, $\mathrm{p}<0.05$ was statistically significant. Differences in mean lesion size, mean age of patients, mean size between groups (malignant/benign) with respect to CEUS result were evaluated by t-test.

215 tumors, 116 (53.93\%) benign and 99 (46.05\%) malignant lesions. 17 (14.66\%) benign and 15 (15.15\%) malignant lesions expressed atypical washout, 5 (4.31\%) benign lesions had early washout and 38 (38.38\%) had continuous washout $(\mathrm{p}<0.0001) .56(48.28 \%)$ benign and $55(55.56 \%)$ malignant lesions had middle degree of opacification, 19 (16.38\%) benign had high and $5(5.05 \%)$ malignant lesions low degree of opacification $(\mathrm{p}<0.0001)$. Finally, $38(32.72 \%)$ benign and 20 (20.20\%) malignant were marked CEUS uncertain. As risk lesions are considered fibroadenomas, fibrocystic lesions, papillomas and low grade DCIS or invasive breast tumors, especially lesions smaller than $10 \mathrm{~mm}$.

Identification of CEUS pitfalls with respect to problematic histopathologic entities can reduce the risk of misdiagnosis and improve further management of breast lesions.
\end{abstract}

Key words: contrast enhanced ultrasound, benign breast tumors, malignant breast tumors, biological dignity

Ultrasound examination belongs to the standard of breast imaging. The assessment of vasculature in breast lesions as one of its important characteristics was recently a role of power and color Doppler mode. This method has its limitations: it is sensitive only to vessels of diameter $\leq 2 \mathrm{~mm}$ what is in case of early stage carcinoma insufficient [1]. During the last decade ultrasound imaging experienced a development of advanced techniques, one of them is contrast enhanced ultrasonography (CEUS).

This method uses intravenous contrast agent with microbubbles and a dedicated software for further analysis. Nowadays used 3rd generation of contrast agents works on the similar principle as breast MRI. It means CEUS reveals the microvascular density of the examined lesion and gives information about perfusion of the tumor [2]. Microbubbles are gas-filled particles with a diameter of several microm- eters. They behave like a hyperechoic oscillating particles that are enhancing the ultrasound signal returning from the examined tissue [3]. This characteristic results in enhancement of the lesions with higher vascular density. Contrast specific software of ultrasound equipment suppresses the signal from the background tissue leaving only the signal from microbubbles (2). In addition, using a high-frequency probe increases the spatial resolution which results in more detailed picture of examined lesion [4]. Contrast enhanced ultrasound provides not only morphological but also functional characteristics of the lesions. The pharmacokinetics of contrast agent is very similar to that of breast MRI: three different ways of washout can be distinguished - continuous, rapid and atypical. The pattern depends on the density of neovasculature which is formed by impaired, tortuous vessels with multiple shunts [5]. 
These bubbles circulate in vessels for couple of minutes until being released from the body by breathing (6). During first minute $40-50 \%$ of contrast agent is eliminated from the circulation, after 10 minutes the residuum is less than $20 \%$. There is no risk of nephrotoxicity, so no renal function blood tests before the examination are needed. Its safety in pregnancy was not proved [6].

Technically, this method has many advantages: fast, tolerated non risk method, without nephron- or hepatotoxicity, with possibility to repeat without any risk, no radiation. On the other side, there are also limitations and pitfalls like movement artifacts, need for experienced radiologist, special dedication to software and also the necessity of peripheral venous access.

Numbers regarding specificity and sensitivity of CEUS differ widely throughout the papers, a wide range of diagnostic performance was found in previously published studies: sensitivity was in the range of $67-100 \%$, while specificity was in the range of $47-100 \%$ [1].

Most of the studies are following the contribution of CEUS in assessment of dignity of lesion. Our prospective study was aimed at the pitfalls of CEUS which can cause differential diagnostic problems. We tried to identify those characteristics of breast lesions that can be the source of diagnostic difficulties and of falsely positive or negative findings. Recognizing these problematic histopathologic entities helps to avoid the misdiagnosis and to improve further management of the patient.

\section{Patients and methods}

Patients. This prospective study was approved by Local Institution Ethics Committee and all patients' informed consent was obtained. From February 2013 to January 2015 215 women (range $23-76$ y, mean age 53.16 y) were examined and included in this study. The patients were selected according to the findings of conventional (non-contrast) ultrasound examination: concluded as BIRADS $4(\mathrm{a}, \mathrm{b}, \mathrm{c})$ or BIRADS 5. The patients in the study had either a solitary lesion or in case of multiple lesions only the largest and the most suspicious ones were examined and biopsied, thus the group of 215 patients formed analyzed set of 215 tumors. The size of the tumor differed - range 3-98 mm, mean $17.98 \mathrm{~mm}$.

All the patients after conventional ultrasound were informed about the need to make histological analysis of the lesions and about the contrast enhanced ultrasound performed prior to the biopsy.

Histopathologic analysis revealed 99 malignant lesions out of which 11 cases were non-invasive malignant lesions, 87 cases were invasive malignant lesions, one was B-NHL and 116 were benign lesions.

Ultrasound technique. The contrast enhanced examinations included in this study were performed using the Philips I U22 ultrasound machine equipped with $12.5 \mathrm{MHz}$ linear transducer and a dedicated software (QLAB - Advanced
Ultrasound Quantification Software release 8.1.2) for further analysis. All examinations were performed by two experienced radiologists with more than 15 years of practice in breast imaging and 4 years in CEUS. Every patient underwent the conventional ultrasound examination to differentiate the suspicious lesion. Than the US system was switched to CEUS with parameters as follows: $0.1-0.4$ for mechanical index, frame rate 5-21 frames/per second. These parameters didn't change during the whole examination. The contrast agent used at CEUS was SonoVue (Bracco Imaging, Milan, Italy) $2.5 \mathrm{ml}$ bolus followed by $5 \mathrm{ml}$ of saline solution.

Image analysis. We used a QLAB - Advanced Ultrasound Quantification Software release 8.1.2 software. In dynamic post contrast study following parameters were analyzed: degree of enhancement according to Echo Mean (dB) (low 0-9.99 dB, middle 10-13.99 dB, high $14 \mathrm{~dB}$ and more, no enhancement), character of contrast agent washout (early $\leq 15 \mathrm{sec}$, continuous, and atypical). As an atypical washout we marked the pattern that didn't fit to two others. According to these parameters, as well as the characteristics of the lesions in $\mathrm{B}$ mode ultrasound, the reader marked every lesion as suspicious (S), non-suspicious (N) or uncertain (U). Suspicious lesions: BIRADS $4 \mathrm{~b}, \mathrm{c}$ or 5 , early washout, middle or high-degree of enhancement. Non-suspicious lesions: BIRADS $4 \mathrm{a}$, continuous washout or no washout and a low degree of enhancement. Uncertain lesions: BIRADS 4 a,b,c, atypical washout, middle or low degree of enhancement.

In every case, CEUS was followed by core cut biopsy, which was performed immediately with withdrawal of 4-5 samples sent for histopathological analysis. Afterwards, the results from biopsy were correlated with the ultrasound characteristics and findings.

Statistical analysis. All tumors were divided into benign and malignant groups with frequency distribution according to their histopathological characteristics. Differences in mean lesion size, mean age of patients as well as differences in mean size between both groups with respect to CEUS result were evaluated by t-test. Qualitative variables such as character of washout, degree of opacification, CEUS results and analysis of CEUS results with respect to histological type were evaluated using Pearson $\chi^{2}$ square test and $p<0.05$ was considered as statistically significant.

For statistical analysis we used software Acastat version 7.0 and StatCalc version 7.1.2, Excel 2010.

\section{Results}

Histologic analysis revealed that from total number of 215 tumors, 116 was of benign character $(53.93 \%)$ and 99 were malignant $(46.05 \%)$. Lesions stratification according to the histopathologic findings is listed in Table 1.

The mean age of patients with benign lesions was $48.03 y$, in the group of malignant tumors the mean age of women was $58.50 \mathrm{y}$. The mean size of malignant lesions was $18.95 \mathrm{~mm}$, of benign tumors $16.53 \mathrm{~mm}(\mathrm{p}=0.009)$. 
First analyzed parameter in the study was the character of washout. 17 out of 116 benign lesions (14.66\%) had atypical character of washout, 94 lesions $(81.03 \%)$ were presented by continuous and 5 lesions $(4.31 \%)$ by early washout. In the group of malignant lesions, 15 out of $99(15.15 \%)$ expressed atypical way of washout, while 38 (38.38\%) had continuous washout and 46 lesions (46.46\%) were characterized by early washout $(\mathrm{p}<0.0001)$. With respect to histology, the presence of atypical washout was statistically significant $(p<0.022)$ : in fibrosclerosis (in 6 cases out of 17 atypically behaving lesions, $35.29 \%$ ), fibrocystic changes (in 5 cases out of 17, 29.41\%), in fibroadenomas ( 3 cases out of 17, 17.65\%) and further entities, as listed in Table 1. Early washout was expressed equally by 1 case out of 5 (20\%) in fibrocystic changes, benign phyllodes tumor, fibroadenoma, chronical inflammation and radial scar and was statistically non-significant (Table 1). According to the histological type in malignant lesion group, atypical washout was detected in 9 cases of IDC out of 15 atypically behaving lesions (60\%), in 3 cases of ILC (20.00\%) and in 2 DCIS (13.33\%) and 1 case (6.67\%) of invasive mucinous carcinoma. The continuous washout appeared in 24 cases of IDC (63.66\%), 7 of DCIS (18.42\% and 3 of ILC out of 38 cases (7.89\%) (Table 1). There was no statistical significance in the presence of atypical washout in malignant and benign group $(\mathrm{p}=0.92)$.

Degree of opacification was the second analyzed sign and was statistically significant $(\mathrm{p}<0.0001)$ (Table 2$)$. The highest number of benign lesions - 56 out of 116 (48.28\%) presented themselves by middle degree $(10-13.99 \mathrm{~dB})$ opacification. 41 lesions (35.34\%) had a low degree (0-9.99 dB)
Table 1. Histopathologic stratification of breast lesions $(n=215)$ and character of washout.

\begin{tabular}{|c|c|c|c|c|}
\hline \multirow{2}{*}{ Histopathological diagnosis } & \multirow{2}{*}{ No } & \multicolumn{3}{|c|}{ Washout } \\
\hline & & Atypical & Continuous & Early \\
\hline Absces & 1 & - & 1 & - \\
\hline Adenosis & 2 & - & 2 & - \\
\hline Fibrocystic changes & 39 & 5 & 33 & 1 \\
\hline Chronical inflammation & 4 & - & 3 & 1 \\
\hline Fibroadenoma & 41 & 3 & 37 & 1 \\
\hline Fibrosclerosis & 15 & 6 & 9 & - \\
\hline Columnar changes & 1 & - & 1 & - \\
\hline Lymphonode & 1 & 1 & - & - \\
\hline Myofibroblastoma & 1 & 1 & - & - \\
\hline Papilloma & 7 & 1 & 6 & - \\
\hline Benign phyllodes tumor & 2 & - & 1 & 1 \\
\hline Radial scar & 2 & - & 1 & 1 \\
\hline DCIS & 11 & 2 & 7 & 2 \\
\hline Invasive ductal carcinoma & 69 & 9 & 24 & 36 \\
\hline Invasive lobular carcinoma & 12 & 3 & 3 & 6 \\
\hline Invasive mixed carcinoma & 1 & - & - & 1 \\
\hline Malignant phyllodes tumor & 2 & - & 2 & - \\
\hline Invasive mucinous carcinoma & 2 & 1 & 1 & - \\
\hline B-NHL nodular & 1 & - & 1 & - \\
\hline Tubulolobular invasive carcinoma & 1 & - & - & 1 \\
\hline Total & 215 & 32 & 132 & 51 \\
\hline
\end{tabular}

and a high degree of opacification (more than $14 \mathrm{~dB}$ ) was observed in 19 out of 116 (16.38\%) lesions. Majority of the malignant lesions were presented by middle degree opacifi-

Table 2. Degree of opacification and CEUS results according to histopathological entities.

\begin{tabular}{|c|c|c|c|c|c|c|c|}
\hline \multirow{2}{*}{ Histological diagnosis } & \multirow{2}{*}{ No } & \multicolumn{3}{|c|}{ Degree of opacification } & \multicolumn{3}{|c|}{ CEUS results } \\
\hline & & Low & Middle & High & Non suspicious & Uncertain & Suspicious \\
\hline Absces & 1 & - & 1 & - & 1 & - & - \\
\hline Adenosis & 2 & - & 1 & 1 & - & 2 & - \\
\hline Fibrocystic changes & 39 & 14 & 17 & 8 & 28 & 10 & 1 \\
\hline Benign phyllodes & 2 & - & 2 & - & 2 & - & - \\
\hline Chronical inflammation & 4 & 1 & 1 & 2 & 2 & 2 & - \\
\hline Fibroadenoma & 41 & 15 & 23 & 3 & 29 & 12 & - \\
\hline Fibrosclerosis & 15 & 10 & 4 & 1 & 9 & 6 & - \\
\hline Columnar changes & 1 & - & 1 & - & - & 1 & - \\
\hline Lymphonodes & 1 & 1 & - & - & 1 & - & - \\
\hline Myofibroblastoma & 1 & - & - & 1 & - & 1 & - \\
\hline Papilloma & 7 & - & 4 & 3 & 4 & 3 & - \\
\hline Radial scar & 2 & - & 2 & - & 1 & 1 & - \\
\hline DCIS & 11 & 1 & 6 & 4 & 2 & 5 & 4 \\
\hline Inv. ductal carcinoma & 69 & 4 & 36 & 29 & - & 11 & 58 \\
\hline Inv. lobular carcinoma & 12 & - & 8 & 4 & - & 3 & 9 \\
\hline Inv. mixed carcinoma & 1 & - & 1 & - & - & - & 1 \\
\hline Malignant phyllodes & 2 & - & 1 & 1 & - & - & 2 \\
\hline Mucinous inv.carcinoma & 2 & - & 2 & - & - & 1 & 1 \\
\hline B-NHL & 1 & - & - & 1 & - & - & 1 \\
\hline Tubulolobular inv. carcinoma & 1 & - & 1 & - & - & - & 1 \\
\hline
\end{tabular}


cation - 55 (55.56\%), while the high degree was observed in 39 lesions (39.39\%) and low degree in 5 (5.05\%). Statistical significance between malignant and benign lesions appeared in case of low and high degree of opacification $(\mathrm{p}<0.0001)$, middle opacification degree was found to be of no significance $(\mathrm{p}=0.23)$ (Table 2.) Histological stratification is listed in Table 2: middle degree was identified in 23 cases of fibroadenomas out of $56(41.07 \%)$, then in 17 cases of fibrocystic changes $(30.36 \%)$ and 4 cases of fibrosclerosis (7.14\%) and in 4 papillomas $(7.14 \%)$. In the group of high degree opacification, 8 lesions $(42.11 \%)$ were defined as fibrocystic changes, $3(15.79 \%)$ as fibroadenoma (Figure 1A) and $3(15.79 \%)$ as papilloma and $2(10.53 \%)$ as chronical inflammation $(\mathrm{p}=0.15)$ (Table 2). Similar analysis revealed that in group of malignant tumors, middle degree opacification was in 36 cases $(65.45 \%)$ of IDC, in 8 cases of ILC (14.55\%) and 6 cases of DCIS $(10.91 \%)$. Low degree opacification appeared in 4 cases of IDC (80\%) (Figure 1B) and 1 of DCIS (20\%) out of 5 lesions $(\mathrm{p}=0.95)$ (Table 2).

According to the washout character and the degree of opacification, each lesion after the CEUS was marked as suspicious (S), non-suspicious (NS) or uncertain (U): from total number of 116 benign lesions 77 (66.38\%) were non-suspicious, $38(32.72 \%)$ were uncertain and $1(0.86 \%)$ was suspicious. From 99 malignant lesions 2 (2.02\%) were marked as non-suspicious, $20(20.20 \%)$ as uncertain and 77 $(77.78 \%)$ as suspicious $(\mathrm{p}<0.0001)$ (Table 2$)$. When regarding "uncertain" marked lesions in both groups as those that were evaluated as falsely positive (in benign group) and false negative (in malignant group), then specificity of CEUS is $85 \%$ and sensitivity $74 \%$. Positive predictive value $85 \%$ and negative predictive value $74 \%$.

With respect to histopathological results in the group of benign lesions with uncertain CEUS outcome, the highest portion was formed by fibroadenomas - 12 out of 38 uncertain lesions (31.58\%, $\mathrm{p}=0.0002)$, followed by fibrocystic changes in 10 cases $(26.32 \%)$ and fibrosclerosis in 6 cases (15.79\%). The only case that was characterized by CEUS as suspicious was finally classified by biopsy as fibrocystic changes (Figure 2A, Table 2).

In the group of 20 malignant lesions marked as CEUS uncertain, 11 (55\%) were invasive ductal carcinomas (IDC), 5 ductal carcinomas in situ (DCIS) (25.00\%) (Figure 2B), 3 invasive lobular carcinomas (ILC) (15.00\%) (Figure 2C) and one mucinous carcinoma (5\%). Two cases of DCIS out of total number of 11 DCIS were characterized as non-suspicious $(18.18 \%)(p=0.03)$ (Figure 2D, Table 2).

When analyzing the CEUS uncertain group of malignant lesions according to the grade of nuclear atypia, we realized that there is no statistical significance $(p=0.35)$ : 9 lesions out of 20 uncertain (45\%) were characterized by histopathological analysis as low-grade lesions, 7 (35\%) as intermediate and $4(20 \%)$ as high-grade lesions. The 2 CEUS non-suspicious lesions were represented by 1 in low-grade group and 1 in intermediate group (Table 3). B-NHL was not included because the grade was not examined.

When dividing malignant and benign lesions into three groups according to the size $(0-10 \mathrm{~mm}, 11-20 \mathrm{~mm}$, more
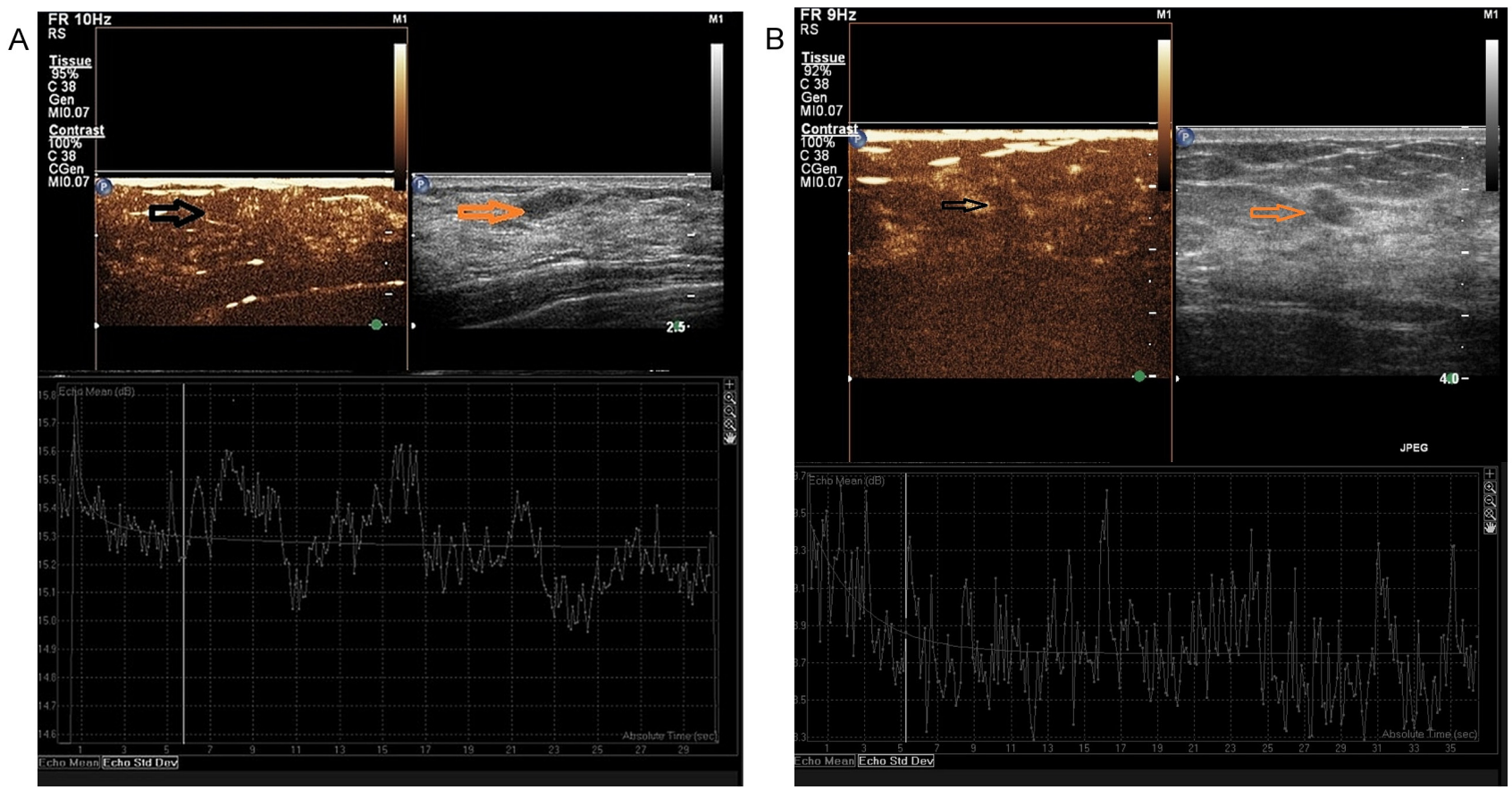

Figure 1. A) fibroadenoma - CEUS uncertain (BIRADS 4a), high degree of opacification (more than $14 \mathrm{~dB}$ ), atypical washout; B) invasive ductal carcinoma grade I - CEUS uncertain (BIRADS 4b), low degree of opacification (less than $9.99 \mathrm{~dB}$ ), atypical washout. 
than $20 \mathrm{~mm}$ ), we realized that the smallest lesions up to $10 \mathrm{~mm}$ appeared as CEUS uncertain almost equally - 15 $(12.93 \%)$ benign lesions vs $12(12 \%)$ malignant $(\mathrm{p}<0.0001)$. When analyzing lesions $11-20 \mathrm{~mm}$, there is $16 \%$ of benign lesions and only $3 \%$ still regarded as CEUS uncertain $(\mathrm{p}<0.0001)$. In the group of lesions bigger than $21 \mathrm{~mm}$, both benign and malignant are regarded as uncertain in almost equal portions $(6 \%$ vs $5 \%)(\mathrm{p}<0.0001)$. The most numerous
Table 3. CEUS outcomes according to the grade of nuclear atypia for malignant breast lesions(B-NHL was not classified according grade of nuclear atypia).

\begin{tabular}{lcccc}
\hline Grade & Nonsuspicious & Uncertain & Suspicious & Total \\
\hline $\mathbf{1}$ & 1 & 9 & 21 & 31 \\
$\mathbf{2}$ & 1 & 7 & 28 & 36 \\
$\mathbf{3}$ & - & 4 & 27 & 31 \\
Total & $\mathbf{2}$ & $\mathbf{2 0}$ & $\mathbf{7 6}$ & $\mathbf{9 8}$ \\
\hline
\end{tabular}
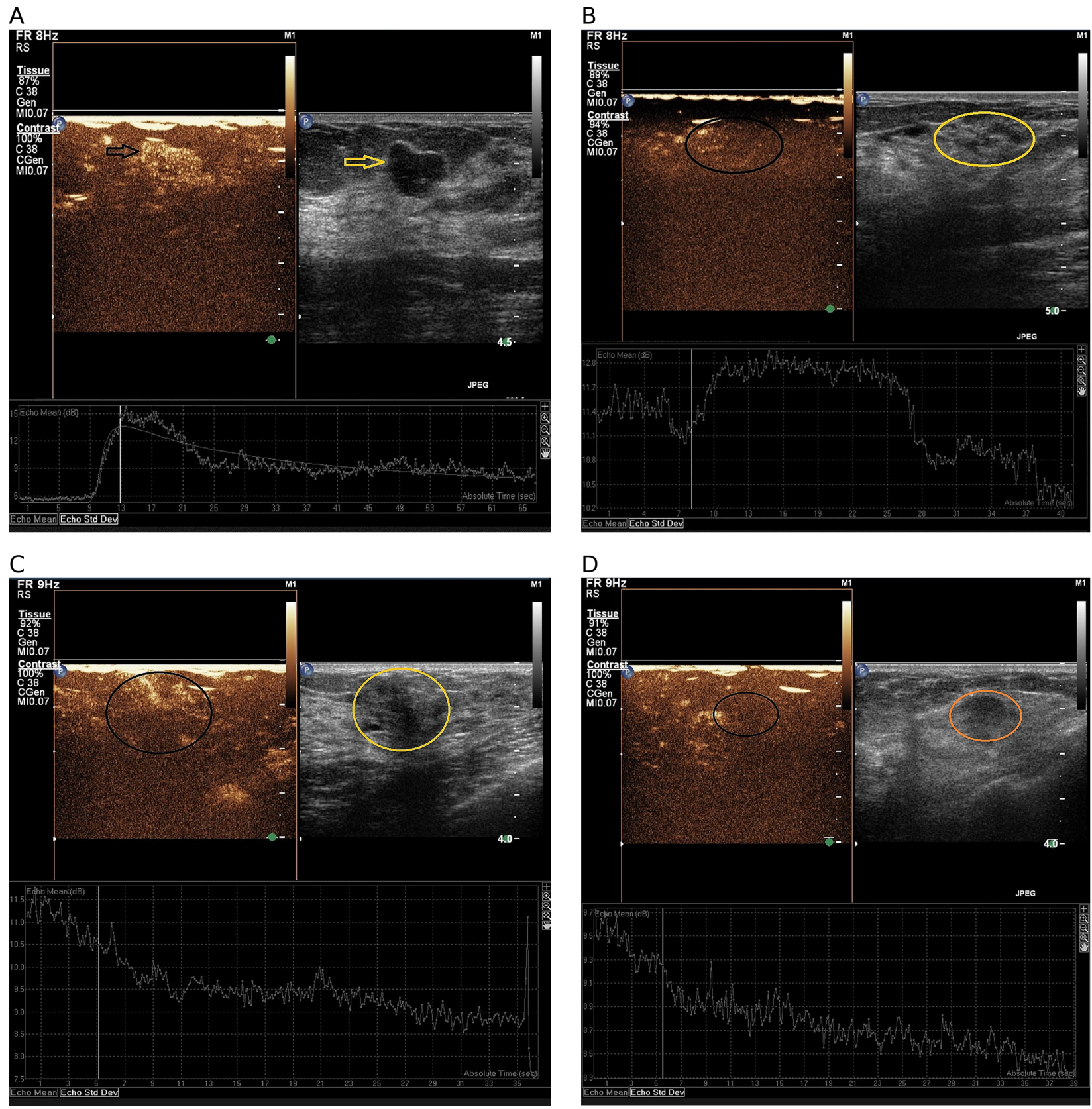

Figure 2. A) fibrocystic changes - CEUS suspicious (BIRADS 4c), high degree of opacification (more than 14dB), early washout; B) ductal carcinoma in situ grade I - CEUS uncertain (BIRADS 4b), middle degree of opacification (up to $14 \mathrm{~dB}$ ), atypical washout; C) invasive lobular carcinoma grade II - CEUS uncertain (BIRADS 4c), middle degree of opacification (10-13.99 dB), atypical washout; D) ductal carcinoma in situ grade I - CEUS nonsuspicious (BIRADS 4a), low degree of opacification (up to $9.99 \mathrm{~dB}$ ), continuous washout. 
Table 4. CEUS outcomes according to the size of the benign/malignant breast lesions.

\begin{tabular}{lccc}
\hline Size of lesion & \multicolumn{3}{c}{ CEUS results } \\
\cline { 2 - 4 } & Non suspicious & Uncertain & Suspicious \\
\hline$<10 \mathrm{~mm}$ & 28 & 15 & 1 \\
Benign & 1 & 12 & 22 \\
Malignant & & & \\
& 24 & 16 & 0 \\
$11-20 \mathrm{~mm}$ & 0 & 3 & 25 \\
Benign & & & 0 \\
Malignant & & 7 & 30 \\
& 25 & 5 & \\
Benign & 1 & & \\
Malignant & & & \\
\hline
\end{tabular}

histologic entity of uncertain lesions up to $10 \mathrm{~mm}$ is DCIS (3 out of 5) and ductal invasive carcinoma (8 out of 69), invasive lobular carcinoma in one case (Table 4$)$.

Among the benign lesions, which are considered as uncertain lesions smaller or equal to $10 \mathrm{~mm}$, we found 6 fibrocystic lesions (out of 39, 15.38\%), 4 fibroadenomas (out of 41, $9.76 \%$ ) and 2 papillomas (out of 7, 28.57\%). Fibroadenomas appeared numerously also in the group of uncertain lesions $11-20 \mathrm{~mm}$ ( 6 out of $41,14.63 \%$ ), followed by fibrosclerosis ( 3 out of $15,0.20 \%$ ) and fibrocystic changes (2 out of $39,5.13 \%$ ) (Table 4).

\section{Discussion}

In recent papers regarding CEUS, the majority of studies are focused on the benefits of this method, on the ability to distinguish between malignant and benign lesions, on predicting prognostic factors of tumors according to the CEUS characteristics or to help to avoid unnecessary biopsies by rerating BIRADS categories [7-9]. Another study proved CEUS to be more accurate than B-mode examination in pre-operative size assessment of invasive ductal carcinoma [10]. In our prospective study we tried to identify the pitfalls and the resulting risks of CEUS. Identifications of those lesions, which present higher risk of over or underestimation, can help to minimize the number of false positive and false negative findings and finally improve the diagnostic potential of this modern method. The next advantage of our study is the number of patients included. 215 patients with equal number of lesions guarantees the statistical relevance in evaluation of parameters and characteristics.

We focused on those lesions that were marked as suspicious or uncertain according to their CEUS parameters, but the histological analysis finally proved them as benign and vice versa. It means we studied benign lesions that expressed atypical or early washout and high degree of opacification. In the group of malignant lesions, our attention was attracted by lesions with "benign behavior" - low degree of opacification and continuous or atypical washout.

Character of enhancement and washout were analyzed also in the study of Jian-Xing Zhang et al. [7] or of Sarracco et al. [8]. Both authors found statistically significant differences in the group of benign and malignant lesions, but with respect to character of enhancement they revealed a group of lesions that might cause diagnostic problems (sensitivity $85 \%$, specificity $85.5 \%$, PPV 75\%, NPV 93\%). We achieved similar results and found out that early washout and high degree of enhancement belong to basic characteristics of malignant tumors. The observations can be explained by the process of tumor neoangiogenesis. It is a development of a new vascular network, which is essential for nutrition of tumor cells and essential to growth and infiltration [7]. According to this fact and similarly to the MRI principle, CEUS has the potential to assess the biological character of breast tumors [11]. With respect to early washout, our outcomes are obvious: it appears in $4.31 \%$ of benign and in $46.46 \%$ of malignant lesions. But our analysis also revealed that there is a substantial overlap between malignant (15.15\%) and benign tumors (14.65\%) when regarding atypical washout. This characteristic appeared almost equally in both groups and the presence was statistically significant $(\mathrm{p}<0.0001)$. The same results were gained when analyzing the degree of enhancement. This parameter correlates also with the density of neovasculature and therefore the high degree of opacification is regarded to be a suspicious mark of the analyzed lesion. Similar to MRI [11], CEUS also revealed that more than half of the malignant lesions (55.56\%) express middle degree of enhancement. In this case, we can see again an overlap with the group of benign lesion. In our study, almost a half of benign tumors (48.58\%) opacificated similarly. From these outcomes it is obvious that there is a statistically significant group of lesions that behaves atypically, what results in "uncertain CEUS conclusion": according to our observation in benign group $32.82 \%$ and in malignant $20.2 \%$.

With the aim to analyze closely these atypically or uncertainly behaving lesions, we correlate the CEUS results with histopathological outcomes. These showed that CEUS has to cope with the same differential diagnostic problems as it is in the case of breast MRI. We see the main pitfall in the benign lesions with higher degree of proliferation and on the other side, in the malignant tumors with lower degree of nuclear atypia. Histopathological analysis of our two groups showed that the most problematic are fibroadenomas together with fibrosclerosis and proliferative fibrocystic changes. These entities usually have very variable and uncertain B-mode ultrasound picture and from our results it is obvious that even CEUS might experience similar problems. These observations are in agreement with outcomes of Huber et al. study, which was aimed at correlation of the histopathologic variability of fibroadenomas and their variable appearance in contrast enhanced power Doppler. They revealed that intratumoral epithelial hyperplasia may contribute to the differen- 
tial diagnostic overlap in some fibroadenomas and thus limit the ability of enhanced power Doppler to distinguish between benign and malignant masses on the basis of enhancement characteristics [12]. Very similar were the preliminary observations of Liu et al. who showed that enhanced areas correlated with invasive carcinoma but also with papillomas and fibroadenomas. He explained these observations by increased cellularity of the lesions as well as by hyperplasia [13].

In the group of malignant lesions, the uncertainty is not so obvious. The highest number was reached by invasive ductal carcinoma ( 11 out of $20,55 \%$ ) followed by DCIS (5 out of $20,20 \%)$. With respect to grade, we found out that DCIS with the uncertain CEUS result had in 2 cases of low nuclear grade and in 3 cases of intermediate, there was no lesion with high grade marked as uncertain. Invasive ductal carcinoma was low grade in 6 cases, in one case intermediate grade and in 4 cases high grade. This distribution was not statistically significant $(\mathrm{p}>0.05)$. It is probably due to the detailed analysis on the level of histopathological unit with respect to nuclear grade which caused the decrease in the size of groups and resulted in non-significance. However, even from these results it is obvious that DCIS, as well as invasive carcinoma with low or intermediate grade, might represent the risk entity with respect to CEUS analysis and that CEUS correlates with qualitative analysis of MR. Very similar conclusions were also drawn in the study Luo et al [14]. They saw the same problem in fibroadenomas which might be the most often falsely positive lesions. On the other side, they evaluated underestimation of malignant lesions to be caused by poor vascularization typical for early grade invasive tumors as well as for the DCIS [14]. The lack of malformed neovascularity, especially in case of low grade DCIS, which depends on normal surrounding vessels for oxygen supply without eliciting abnormal vessel generation, can lead to misdiagnosis and underestimation of these malignant lesions [15]. Specific was invasive lobular carcinoma. When analyzing grade of all 12 lesions, we found out that they were exclusively of low or intermediate grade. Poor neovascularization together with specific growth pattern makes this entity a diagnostic challenge [16].

Finally, we analyzed the size of tumors in both groups (Table 4). From the gained results it is obvious that smaller lesions could cause diagnostic problem. The problem with small lesions and CEUS examination tried to explain in their study Zhao et al. They reported that lesions with different size expressed different CEUS features that are dependent on the neovascularisation [17], and this can cause a diagnostic ambiguity. Small lesions according Zhao (smaller than $20 \mathrm{~mm}$ ) might have poor neovascularization due to slow growth, low grade or early stage of disease. This correlates with our results. In the group of the lesions smaller or equal to $10 \mathrm{~mm}$, we found out that benign and malignant lesions were equally presented as uncertain, while in the malignant group there were almost exclusively low grade and intermediate grade lesions. There is no relationship between the size of ILC and CEUS results, there were 3 uncertain invasive lobular carcinomas distributed evenly in all three groups. This could be explained by the different pattern of spreading of ILC which is more infiltrating. The borders of the lesion are obscured and difficult to differentiate. Specific histopathologic features of ILC seem to be translated into their qualitative sonographic appearance [18].

In conclusion, CEUS as the very promising method has the potential to differentiate between benign and malignant lesions according to the degree of opacification and character of washout, which are functional characteristics of the lesion. This makes CEUS superior to mammography and conventional ultrasonography, which are the basic non-invasive methods used in breast lesions diagnostics. These methods provide only morphologic characteristics and often there is an overlap between benign and malignant breast lesions. Similarly to MRI, also when using CEUS we can see some pitfalls that are caused by histopathologic character of lesion and which are the source of diagnostic ambiguity. These might result in falsely positive or negative conclusions. The most problematic in the benign group seem to be fibroadenomas and proliferative fibrocystic changes together with papillomas. Between malignant lesions, we still see the problem in low grade DCIS and low grade invasive tumors, especially regarding lesions smaller than $10 \mathrm{~mm}$. These observations result in conclusion that biopsy still remains the method of choice especially in case of early breast cancer.

\section{References}

[1] HU Q, WANG XY, ZHU SY, KANG LK, XIAO ZJ et al. Meta-analysis of contrast-enhanced ultrasound for the differentiation of benign and malignant breast lesions. Acta Radiol 2015; 1: 25-33. doi: 10.1177/0284185113517115

[2] WILSON SR, BREENBAUM LD, GOLDBERG BB. Contrast Enhanced Ultrasound: What is the Evidence and What Are the Obstacles? AJR Am J Roentgenol 2009; 193: 55-60. doi: 10.2214/AJR.09.2553

[3] SUNIL U, KLIBANOV AL. Microbubbles as Ultrasound Contrast Agents for Molecular Imaging: Preparation and Application. AJR Am J Roentgenol 2012; 199: 292-299. doi: 10.2214/AJR.12.8826

[4] XIA HS, WANG X, DING H, Wen JX, FAN PL et al. Papillary breast lesions on contrast-enhanced ultrasound: morphological enhancement patterns and diagnostic strategy. Eur Radiol 2014; 24: 3178-3190. doi: 10.1007/s00330-014-3375-7

[5] YUAN Z, QUAN J, YUNXIAO Z, JIAN C, ZHU H et al. Diagnostic value of Contrast-Enhanced Ultrasound Parametric Imaging in Breast Tumors. J Breast Cancer 2013; 16: 208213. doi: 10.4048/jbc.2013.16.2.208

[6] JANG JY, KIM MY, JEONG SW, KIM TY, KIM SU et al. Current consensus and guidelines of contrast enhanced ultrasound for the characterization of focal liver lesions. Clinical and molecular hepatology 2013; 19: 1-16. doi: 10.3350/ cmh.2013.19.1.1 
[7] ZHANG J, CAI L, CHEN L, DAI J, SONG G. CEUS Helps to Rerate Small Breast Tumors of BI-RADS Category 3 and Category 4. BioMed Res Int 2014; 2014: 572532. doi: $\underline{10.1155 / 2014 / 57253}$

[8] SARACCO A, SZABO BK, ASPELIN P, LEIFLAND K, WILCZEK B et al. Differentiation between benign and malignant breast tumors using kinetic features of real-time harmonic contrast-enhanced ultrasound. Acta Radiol 2012; 53: 382388. doi: $10.1258 /$ ar.2012.11056

[9] MIYAMOTO Y, ITO T, TAKADA E, OMOTO T, HIRAI F et al. Efficacy of Sonazoid (Perflubutane) for contrastenhanced ultrasound in the differentiation of focal breast lesions: phase 3 multicenter clinical trial. AJR Am J Roentgenol 2014, 202: W400-W407. doi: 10.2214/AJR.12.10518

[10] VAN ESSER S, VELDHUIS WB, VAN HILLEGERSBERG R, VAN DIEST PJ, STAPPER G et al. Accuracy of contrast enhanced breast ultrasound for pre-operative tumor size assessment in patients diagnosed with invasive ductal carcinoma of the breast. Cancer Imaging 2007; 7: 63-68.

[11] CAPRONI N, MARCHISIO F, PECCHI A, CANOSSI B, BATTISTA $\mathrm{R}$ et al. Contrast-enhanced ultrasound in the characterisation of breast masses: utility of quantitative analysis in comparison with MR. Eur Radiol 2010; 20: 1384 -1395. doi: 10.1007/s00330-009-1690-1

[12] HUBERS, VESELY M,ZUNA I, GELOME S, CZEMBIREKH. Fibroadenomas: (Computer-assisted quantitative evaluation of contrast-enhanced power Doppler features and correlation with histopathology). Ultrasound Med Biol 2001; 27: 3-11.
[13] LIU H, JIANG YX, LIU JB, ZHU QL, SUN Q et al. Contrastenhanced breast ultrasonography: imaging features with histopathologic correlation. J Ultrasound Med 2009; 28: 911-920.

[14] LUO J, CHEN JD, CHEN Q, YUE LX, ZHOU G et al. Contrast-enhanced ultrasound improved performance of breast imaging reporting and data system evaluation of critical breast lesions. World J Radiol 2016; 8: 610-617. doi: 10.4329/ wjr.v8.i6.610

[15] XIAO X, OU B, YANG H, WU H, LUO B. Breast contrastenhanced ultrasound: Is a scoring system feasible? A preliminary study in China. PLoS ONE 2014; 9: e105517. doi: 10.1371/journal.pone.0105517

[16] McCART REED AE, KUTASOVIC JR, LAKHANI SR, SIMPSON PT. Invasive lobular carcinoma of the breast: morphology, biomarkers and 'omics. Breast Cancer Res 2015; 17: 12. doi: 10.1186/s13058-015-0519-x

[17] ZHAO LX, LIU H, WIE Q, XU J, WU J et al. Contrast enhanced ultrasonography features of breast malignancies with different sizes: Correlation with different prognostic factors. Biomed Res Int 2015; 2015: 613831. doi: $\underline{10.1155 / 2015 / 613831}$

[18] BRKLJACIC B, DIVJAK E, TOMASOVIC-LONCARIC C, TESIC V, IVANAC G. Shear-wave sonoelastographic features of invasive lobular breast cancers. Croat Med J 2016; 57: 42-50. 